\title{
Shear-Strain Step Response in Linear Regime of Dilute Suspensions of Naturally Bent Carbon Nanotubes
}

\author{
C. Cruz $_{,}{ }^{1}$ L. Illoul, ${ }^{1}$ F. Chinesta, ${ }^{2}$ G. Régnier ${ }^{1}$ \\ ${ }^{1}$ Laboratoire des Procédés et Ingénierie en Mécanique et Matériaux (PIMM, UMR CNRS 8006), \\ Arts et Métiers ParisTech, 151 bd de l'Hôpital, 75013 Paris, France \\ ${ }^{2}$ EADS Corporate International Chair, Ecole Centrale de Nantes, 1 rue de la Noë-BP 92101, \\ 44321 Nantes Cédex 3, Nantes, France
}

\begin{abstract}
Impressive enhancements of the storage modulus have been documented when low volume fractions of single wall carbon nanotubes (SWNTs) are added to a Newtonian solvent for obtaining dilute suspensions. The intrinsic bending dynamics of carbon nanotubes (CNTs) has been proposed to explain such elasticity. CNTs contain topological defects inducing naturally bent structures in absence of external forces and, hence, a semiflexible filament with a bent configuration at minimal internalbending-energy is used for mimicking the structure of SWNTs in suspension. Previous continuous model is discretized as a nonfreely jointed bead-rod chain with a naturally bent configuration for simulating the rheological behavior after a shear-strain step in linear regime of
\end{abstract}

SWNT dilute suspensions by using a Brownian dynamics (BD) approach. In general, bead-rod chains exhibit an instantaneous relaxation after a high shear-strain step. Bending rigidity and number of constitutive rods are found to be determinant parameters in the internal-energy relaxation behavior of nonfreely jointed bead-rod chains in dilute solution. Proper comparisons between the BD simulation results and the experimental data for treated SWNT dilute suspensions confirm the consistency of the physical model mimicking the structure of a SWNT. (c) 2012 Wiley Periodicals, Inc. J Appl Polym Sci 000: 000-000, 2012

Key words: carbon nanotubes; dilute suspension; linear viscoelasticity; shear relaxation; Brownian dynamics

\section{INTRODUCTION}

A carbon nanotube (CNT) is an allotropic form of carbon with a cylindrical structure constituted by rolled-up graphene sheets with diameters at the nanoscale $(0.4-50 \mathrm{~nm})$ and lengths at the micro to macroscale. Particularly, a SWNT is made just by one rolled-up graphene lattice. In addition to their unique structure, interest for CNTs is based on their impressive mechanical, ${ }^{1-4}$ thermal, ${ }^{5}$ and electrical ${ }^{6,7}$ properties. CNTs have been envisaged as component-material in a large range of applications: electrical circuits, 8,9 nanoprobes for near-field microscopes, ${ }^{10}$ high-resolution gas-molecules detectors, ${ }^{11}$ drug and gene delivery systems, ${ }^{12,13}$ high-strength fibres, ${ }^{14}$ and high-performance polymer-based composites. ${ }^{15-17}$ Many of the previously mentioned applications of CNTs require a transformation processing in liquid phase. Even more, purification and chemical modification of CNTs usually happen in solution. For such reason, CNTs are usually suspended into a Newtonian solvent or a viscoelastic

Correspondence to: G. Régnier (gilles.regnier@ensam.eu). matrix (e.g., melted polymer). Control of those forming processes needs a deep understanding of the rheology of CNT suspensions. ${ }^{18}$ Physical properties of CNT suspensions are controlled by many factors, for example, the number of walls, chirality, purity, defects, dimensions and surface treatment of the nanotubes, CNT volume fraction, suspending medium, morphologies, and dispersion states. ${ }^{19}$ As a consequence, a vast range of flow phenomena in CNT suspensions has been documented due to the large variability of the factors controlling the physical properties of CNT suspensions. Nevertheless, the increasing interest on the processing and the potential applications of those suspensions has revealed a lack of coupling between experimental and computational rheology. ${ }^{20}$ In response, this article deals with theoretical rheology looking for determining the role of the intrinsic CNT structure (bending flexibility, defects) in the deformation mechanics of CNT suspensions. In such context, rheological behavior of dilute and low semidilute suspensions of treated SWNT within a Newtonian solvent constitutes a suitable framework for studying the dynamics of individual CNTs in suspension.

CNTs have an important influence on the viscous behavior of their suspensions, even for dilute suspensions. ${ }^{21}$ Dilute and low semidilute suspensions of treated SWNT $(0.05,0.1$, and 0.2 wt \%) within an 
epoxy resin exhibit a typical shear-thinning behavior. Given that treated SWNTs are side-wall chemically modified, agglomeration is largely prevented and treated SWNTs are supposed to be suspended mainly in the form of individual nanotubes. Shearthinning behavior has been explained in terms of the competition between two physical phenomena: SWNT orientation in the flow direction and SWNT random misalignment due to Brownian motion (thermal interaction with the solvent). In fact, shearthinning signature has been satisfactorily modeled by a Fokker Planck (FP) simple orientation model. In that modeling, SWNTs were considered as rigid fibres with high aspect ratio $(\sim 200)$, subjected to a homogeneous shear-flow and rotary thermal diffusion. ${ }^{22}$ Consistency of the FP-orientation model validated the hypothesis of the nanotube alignment in the shear direction as the main physical phenomenon explaining the shear thinning behavior exhibited by dilute treated-SWNT suspensions. Moreover, shearinduced alignment of CNT has been proved experimentally before. ${ }^{21,23}$ Given the high aspect ratio and tensile rigidity of CNTs, the classical mesoscopicmacroscopic approaches for fibre suspensions ${ }^{24-27}$ can also be employed to describe the rheological behavior of CNT suspensions. However, use of closure relations for the orientation tensors, which are only exact for a restricted number of microstructures, can negatively influence the numerical solutions. ${ }^{28,29}$ Using a morphological-based approach, shear-thinning behavior of treated sonicated carbon nanofiber aqueous suspensions was correctly modeled. An elastic dumbbell model, whose parameters were determined from a morphological characterization by SEM, was able to capture the steady shear behavior of treated sonicated carbon nanofiber aqueous suspensions in dilute and low semidilute regime. ${ }^{30}$ In fact, some CNTs have similar dimensions than carbon nanofibers (diameter of about 100-200 nm) and, in consequence, microstructures of both dilute suspensions can be considered as analogues.

When dilute suspensions of treated SWNT (0.1 and $0.2 \mathrm{wt} \%$ ) are submitted to a small-amplitude oscillatory deformation a nonnegligible elastic dynamic response is identified in the tested frequency range. Contribution of the SWNTs to the storage and loss modulus exhibits a constant-slope evolution in the tested frequency range. Several hypotheses for explaining this interesting linear viscoelastic response have been postulated as, for example, the existence of a weak SWNT network, ${ }^{31}$ the electrostatic interactions between surface-treated SWNTs $^{21}$ and the bending dynamics of individual SWNTs $^{32}$; however the debate about the physical origins of this mild elasticity in the linear viscoelasticity behaviour of dilute SWNT suspensions remains an open question. Those complex modulus data were fitted using a FP-based orientation model (satisfactory in the steady shear scenario), but using an empirical relationship for the rotary diffusion coefficient that depends on the applied frequency. ${ }^{21}$ This means that a simple rigid-rod model is not enough to explain the dynamics of individual SWNTs in suspension and that a more detailed physical description is required to explain the flow phenomena observed in dilute SWNT suspensions. Rheological behavior of CNT suspensions is supposed to be strongly influenced by the recoverable bending of the CNT structure. ${ }^{20}$ For instance, it has been conjectured that an elastic dumbbell model is more successful than a rigid dumbbell model for capturing the linear viscoelastic response of treated sonicated carbon nanofiber suspensions given the fact that the former model can incorporate the recoverable bending of the individual carbon nanofibers. ${ }^{30}$ Nevertheless, such phenomenological modeling is difficult to justify from a physical point of view. Structure of CNTs has been recurrently mimicked as a mesoscale parallel of the structure of rigid rod-like polymer molecules, ${ }^{20,33-35}$ usually modeled as semiflexible filaments or worm-like chains. ${ }^{36}$ In fact, Brownian dynamics (BD) simulations of small-amplitude dynamical solicitations on dilute solutions of semiflexible chains have showed a mild elastic response in the intermediate and high frequency range. ${ }^{37}$ In addition, considering the probable existence of topological bent defects on the CNT structure, BD simulations of small-amplitude oscillatory deformation tests on dilute suspensions of SWNTs (mimicked by semiflexible chain models with a natural bent configuration) demonstrated that nonnegligible differences exist with respect to the dynamic response of dilute suspensions of semiflexible chains with naturally straight configurations. ${ }^{38}$ The assumption of the natural existence of bent junctions in the CNT structure is justified by two facts:

- The common apparition of topological defects (for instance, pentagon/heptagon pair defect) during the CNT synthesis ${ }^{39}$ that can induce several kinds of curvatures on the CNT structure according to the specific morphology of the localized defect. ${ }^{40-45}$

- The experimental evidence obtained by Fresnel projection microscopy, ${ }^{46}$ scanning tunnelling microscopy (STM), ${ }^{47,48}$ voltage-contrast scanning electron microscopy (VC-SEM), ${ }^{49}$ and atomic force microscopy $(\mathrm{AFM})^{50}$ revealing both the presence of localized junctions on CNT structures and a generalized tortuosity in CNT samples.

In front of so impressive rheological behavior of CNT suspensions, one can also be interested in how stresses evolve after applying a small-amplitude 


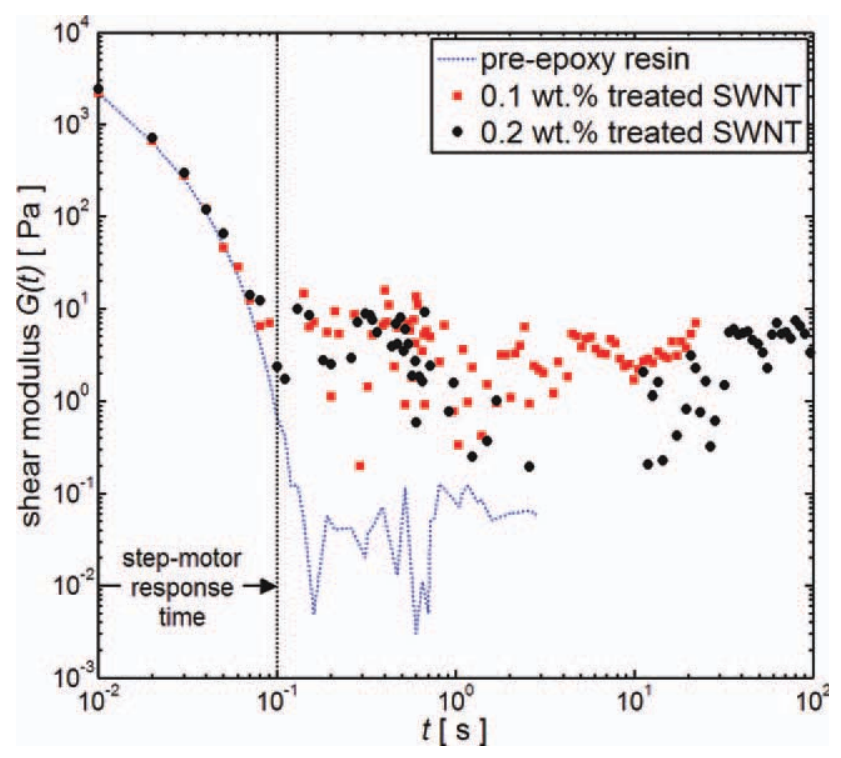

Figure 1 Shear modulus of an epoxy resin and two different dilute suspensions of treated SWNT within an epoxy resin. Response after a $1 \%$ shear-strain step. Isothermal tests at $25^{\circ} \mathrm{C}$. Courtesy of Dr. Anson Ma (Rice University). [Color figure can be viewed in the online issue, which is available at wileyonlinelibrary.com.]

shearing step. Shear-stress relaxation tests are intended to provide additional information about the spectra of characteristic-times associated with the mechanical response of a viscoelastic system during a transient state. Nevertheless, contrarily as expected, a viscoelastic effect in dilute SWNT suspensions is very difficult to distinguish after a shearstrain step in the linear regime, as noticed in Figure 1. So, to evaluate the consistency of the semiflexible chain model with natural bent configuration for mimicking the structure of treated SWNTs, this work proposes to simulate the rheological response of dilute SWNT suspensions after a shear-strain step in the linear regime by using a BD-based stochastic approach. The body of this numerical article is divided in four sections. The most important aspects of the BD modeling of CNT dilute suspensions are presented in Modeling section and the main validation issues of the employed BD approach are detailed in BD Modeling Validation section. Subsequently, in Shear Relaxation Test Algorithm section, the $\mathrm{BD}$ algorithm for simulating a shear relaxation test is described and, finally, the numerical results are discussed and compared with an experimental reference (Fig. 1) in Results and Discussion section.

\section{MODELING}

Longitudinally inextensible high aspect-ratio systems in solution can be represented as rigid rods or semiflexible filaments in function of the persistence length $\left(L_{p}\right)$ associated with. ${ }^{36,37}$ In absence of external forces (e.g., an external flow field), $L_{p}$ establishes a length scale from which the high aspect-ratio system exhibits significant curvature due to the thermal interactions coming from the solvent molecules. Persistence length of SWNTs approaches its physical length $L\left(L_{p} \sim L\right)$ in absence of external forces and/or under the action of an external flow field ${ }^{51,52}$ and, hence, can be represented by semiflexible filaments. In addition, we are tackling with slender systems exhibiting a bent structure in absence of external forces due to the existence of structural defects (e.g., topological defects on the honeycomb-like lattices of CNTs). ${ }^{39-45}$ Previous physical model can also be extended for representing the structure of stiff macromolecules with sterical-hindered dihedral angles as short DNA, collagen fibrils, rod-like viruses and some synthetic polymers. ${ }^{32}$

To simulate the rheological behavior after a shearstrain step applied on a SWNT dilute solution a BD approach is employed. Semiflexible filament model is hence discretized in a nonfreely jointed bead-rod chain model, as showed in Figure 2, to implement the BD simulation. This bead-rod chain model is composed of $n$ beads with positions $\mathbf{r}_{i}$, connected by $n$ - 1 rods of length $a$, where $a$ is close to the system persistence length under flow conditions. Physical length of the SWNT is given by the total length of the bead-rod chain $(n-1) a$. Beads are considered the centres of hydrodynamic resistance, as usual for all the mechanical models in polymer kinetic theory, ${ }^{53}$ and also represent the existence of a structural bent junction. On the other hand, rods are supposed infinitely rigid. Bending flexibility is considered by introducing an internal bending potential, given by the action of a hypothetical flexion spring between each pair of rods. Rod misalignment (i.e., bent structure in absence of external forces) is generated following the next formula:

$$
\theta_{i}^{0}=\theta_{i-1}^{0}-\Delta \theta^{\max }+U(0,1) \cdot 2 \Delta \theta^{\max }
$$

where $\theta_{i}^{0}$ is the minimal-bending-energy director angle of rod $i, \Delta \theta^{\max }$ is the maximal misalignment between two consecutive rods and $U(0,1)$ is a

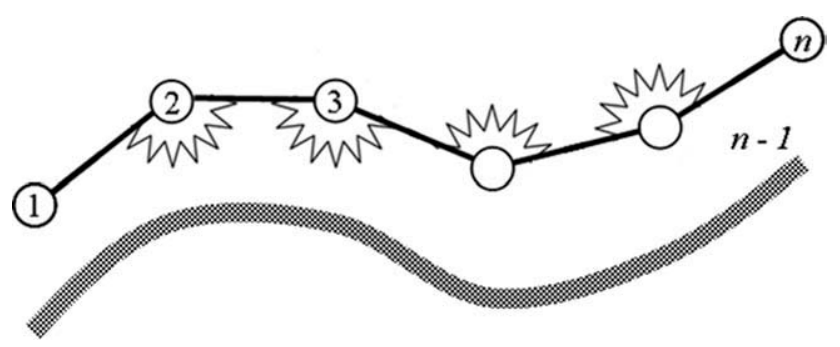

Figure 2 Nonfreely jointed bead rod chain model composed of $n$ beads and $n-1$ rods of length $a$. Bending potential between rods in the bead rod chain is mimicked with a hypothetical flexion spring. An equivalent continuous semiflexible filament is depicted below the discritized model. 
continuous uniform random distribution function defined between 0 and 1 .

In the light of the previous discretization two nonnegligible assumptions have been introduced: (1) bent junctions are homogeneously distributed along the main axis of the nanotube and (2) bent junctions are essentially the active flexion points in the SWNT structure. As we are dealing with systems whose persistence length is close to its physical length $\left(L \sim L_{p}\right)$, the bead-rod chains studied in this article are restricted to a low number of constitutive rods $(n<10)$. Current modeling approach supposes also an isotropic friction tensor. On the other hand, BD simulation of a rheological test in the linear regime of a dilute suspension of SWNTs is subjected to the next hypotheses: (1) tests are carried out at constant temperature, (2) nanotubes are embedded into a Newtonian matrix of viscosity $\eta_{s}$, (3) kinematics of the Newtonian matrix is considered undisturbed by the presence of nanotubes (acceptable assumption in dilute regime and under rheometric flows), (4) applied flow field is considered homogeneous at the scale of the nanotube (i.e., the rate-of-strain tensor is supposed constant at length scales lower than twofold the contour length of the SWNT), (5) physical interactions between nanotubes are neglected due to the dilute regime, (6) hydrodynamic interaction is neglected due to the relative high bending stiffness of SWNTs, and (7) electrostatic, magnetic, and gravitational external forces are not taken into account.

\section{Kinematic and dynamic formulation}

Given the inextensibility condition imposed on the constitutive rods, the bead-rod chain is submitted to the next $n-1$ kinematic restrictions:

$$
\left(\dot{\mathbf{r}}_{i+1}-\dot{\mathbf{r}}_{i}\right) \cdot \mathbf{u}_{i}=0
$$

where $\dot{\mathbf{r}}_{i}$ is the instantaneous velocity of bead $i$ and $\mathbf{u}_{i}$ is a unit vector collinear to the rod connecting beads $i$ and $i+1$ :

$$
\mathbf{u}_{i}=\frac{\left(\mathbf{r}_{i+1}-\mathbf{r}_{i}\right)}{a}
$$

Kinematic evolution of the bead-rod chain model in a Cartesian coordinates system is then well defined by the resolution of $n$ stochastic equations establishing the force balance on each bead of the system:

$$
\mathbf{0}=\mathbf{F}_{i}^{(d)}+\mathbf{F}_{i}^{(b)}+\mathbf{F}_{i}^{(\phi)}-\sum_{j} \mathbf{n}_{i j} \lambda_{j}
$$

where $\mathbf{F}_{i}^{(d)}$ is the hydrodynamic drag force acting on bead $i, \mathbf{F}_{i}^{(b)}$ is the Brownian force acting on bead $i$, $\mathbf{F}_{i}^{(\phi)}$ is the internal bending potential force acting on bead $i$ and $\lambda_{j}$ is the module of the rod tension emerging on bead $j$ for maintaining the distance between beads $j$ and $j+1$ at a constant value $a$. Operator $\mathbf{n}_{i j}$ is defined as follows:

$$
\mathbf{n}_{i j}=\mathbf{u}_{j}\left(\boldsymbol{\delta}_{i j+1}-\boldsymbol{\delta}_{i j}\right)
$$

where $\boldsymbol{\delta}_{i j}$ is the Kronecker delta.

Hydrodynamic drag force acting on bead $i, \mathbf{F}_{i}^{(d)}$, is given by:

$$
\mathbf{F}_{i}^{(d)}=\zeta\left(\left[\mathbf{\kappa} \cdot \mathbf{r}_{i}\right]-\dot{\mathbf{r}}_{i}\right)
$$

where $\zeta$ is the bead friction coefficient and $\boldsymbol{\kappa}$ is the gradient of the external velocity field. Brownian forces, for unconstrained systems, are given by a normal distribution characterized by next moments:

$$
\begin{gathered}
\left\langle\mathbf{F}_{i}^{(b)}(t)\right\rangle=\mathbf{0} \\
\left\langle\mathbf{F}_{i}^{(b)}(t) \otimes \mathbf{F}_{i}^{(b)}(t+\Delta t)\right\rangle \approx \frac{2 \zeta k_{B} T}{\Delta t} \boldsymbol{\delta}
\end{gathered}
$$

where $k_{B}$ is the Boltzmann constant, $T$ is the absolute temperature, $\Delta t$ is the time step of the simulation. In the case of systems containing physical constraints a geometrical projection is executed on the unconstrained Brownian forces for satisfying the fluctuation dissipation theorem:

$$
\sum_{i} \mathbf{F}_{i}^{(b)} \cdot \mathbf{n}_{i j}=0
$$

The algorithm employed to verify (9) has been detailed before. ${ }^{32}$ In accord with the worm-like chain model for semiflexible polymers, ${ }^{36}$ the internal bending potential can be redefined for a bead-rod chain model with nonstraight minimal bending-energy configuration:

$$
\phi=-\frac{K_{b}}{a} \sum_{i=2}^{n-1} \mathbf{Z}_{i} \mathbf{u}_{i} \cdot \mathbf{u}_{i-1}=-\frac{K_{b}}{a} \sum_{i=2}^{n-1} \mathbf{u}_{i}^{\prime} \cdot \mathbf{u}_{i-1}
$$

where $\phi$ is the bending potential, $K_{b}$ is a bending rigidity constant, $\mathbf{Z}_{i}$ is a linear operator that counter-rotates vector $\mathbf{u}_{i}$ of $\Delta \theta_{i, i-1}^{0}$ (internal angle between $\mathbf{u}_{i}$ and $\mathbf{u}_{i-1}$ at the minimal bending-energy configuration) and $\mathbf{u}_{i}^{\prime}$ is the rotated vector $\mathbf{Z}_{i} \mathbf{u}_{i}$. Bending force acting on each bead can then be determined directly as follows:

$$
\mathbf{F}_{k}^{(\phi)}=-\frac{\partial \phi}{\partial \mathbf{r}_{k}}=\frac{K_{b}}{a} \sum_{i=2}^{n-1} \frac{\partial\left(\mathbf{u}_{i}^{\prime} \cdot \mathbf{u}_{i-1}\right)}{\partial \mathbf{r}_{k}}
$$

An algorithm for calculating the bending forces in coherence with a naturally bent configuration and 
based on the local character of the internal bending potential given in (10) has been detailed elsewhere. ${ }^{38}$

In summary, using the $n-1$ equations given in (2) plus the $n$ equations given in (4) a linear system of equations describing the motion of the bead-rod chain model can be established, where the unknown variables are $n$ instantaneous bead velocities $\dot{\mathbf{r}}_{i}$ plus $n-1$ rod tensions $\lambda_{j}$. Position of the bead-rod chain model at the end of a time step is calculated using the bead velocities determined at the beginning of the time step through a first-order integration scheme guaranteeing constant rod lengths during the entire time step. Such single-step algorithm is coherent with the explicit corrector-predictor integration scheme $e^{54,55}$ given the fact that the velocity of each rod during an entire time step is always perpendicular to its orientation. ${ }^{56}$

\section{Evaluation of the stress tensor}

Total shear stress tensor $\tau$ in the suspension can be considered as the sum of two contributions, one coming from the solvent $\tau_{s}$ and another one coming from the suspended particles $\tau_{p}^{53}$ :

$$
\tau=\tau_{s}+\tau_{p}
$$

Shear stress tensor contribution coming from the Newtonian solvent can be written as $\tau_{s}=\eta_{s} \dot{\gamma}$, where $\eta_{s}$ is the solvent viscosity and $\dot{\gamma}$ is the homogeneous rate-of-strain tensor. On the other hand, to quantify the shear stress contribution coming from the suspended particles (i.e., SWNTs represented as nonfreely jointed bead-rod chains) in a BD framework we resort to the classical Kramers-Kirkwood expression for coarse-grained models in kinetic theory:

$$
\tau_{p}=c \sum_{v}\left\langle\mathbf{R}_{v} \otimes \mathbf{F}_{v}^{(d)}\right\rangle
$$

where $c$ is the number of suspended model chains per volume and $\mathbf{R}_{v}=\mathbf{r}_{v}-\mathbf{r}_{c}$ is the relative location of bead $v$ to the centre of mass of the multi bead-rod chain $\mathbf{r}_{c}$.

Special care must be taken when calculating numerically the shear stress tensor for model systems containing constraints. Given the numerical singularities originated by the presence of constraints, particular attention is required for implementing correctly the Kramers-Kirkwood shearstress formula into the $\mathrm{BD}$ algorithm. It has been emphasized that a correct estimation of the shearstress tensor must to be closely linked to the timeintegration scheme used in the simulation. ${ }^{56,57}$ In fact, for evaluating rigorously the Brownian contribution to the shear-stress tensor the implemented algorithm must to consider the discontinuity of the
Brownian forces at each time step, especially when no-flow conditions are being simulated. A "longlasting" effect of the instantaneous random forces generated at the beginning of each time step is considered here for calculating correctly expression (13) inside a first-order integration scheme. Envisaged algorithm is composed of three steps:

1. Update the bead positions by executing one explicit time step from $t$ to $t+\Delta t$.

2. Using the same geometrically projected Brownian forces obtained at time $t$, recalculation of the bead velocities and hydrodynamic drag forces $\tilde{\mathbf{F}}_{i}^{(d)}(t+\Delta t)$ using the updated configuration at $t+\Delta t$.

3. Determination of the shear-stress tensor at $t$ by computing:

$$
\tau_{p}(t)=c \sum_{v}\left\langle\mathbf{R}_{v}(t+\Delta t) \otimes \tilde{\mathbf{F}}_{i}^{(d)}(t+\Delta t)\right\rangle
$$

\section{BD MODELING VALIDATION}

Current BD routine was programmed on $\mathrm{C}++$, compiled in Linux 64-bits and run on a cluster 6× QuadCore AMD Opteron ${ }^{\mathrm{TM}} 2376(2300 \mathrm{MHz})$ processor. Present BD modeling was validated before in front of the linear viscoelastic response of a dilute solution of freely jointed (three-bead)-(two-rod) chains, also known as trimer systems. ${ }^{38}$ Exact dynamic response of a dilute solution of trimer systems was determined by Hassager. ${ }^{58}$ Using the previously described BD modeling in a bidimensional framework, trimer system was simulated by neglecting the internal bending potential (i.e., $K_{b}=0$ ). Complex modulus obtained by the current BD simulation was found in good agreement with Hassager's solution for the complex modulus. ${ }^{38}$ As bidimensional modeling was correctly validated, this work has employed the same dimensionality for the sake of reducing the simulation times.

An analysis of the performance of the current BD modeling in dynamic mode established that a BD population of $10^{4}$ trimers offers a good compromise between computational time and convergence to the central values for an extended reciprocal-frequency test going from $\omega \lambda_{3}=10^{-2}$ to $\omega \lambda_{3}=10^{2}$, where $\lambda_{3}$ is the rotational diffusion time of an equal length (3beads) rigid rod system. ${ }^{38}$ However, given the fact that the solicitation regime simulated in the current article is analogue with that one found in a dynamical test at high frequencies, perturbations coming from the random number generation are strongly reduced and, hence, a BD population of 5000 entities is enough to obtain convergence to the central values. On the other hand, considering that a first-order 


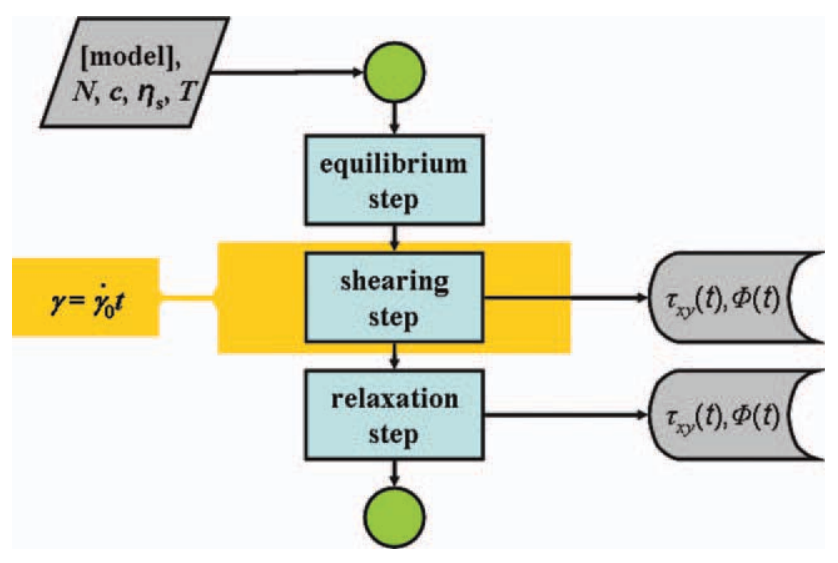

Figure 3 Flowchart of the general BD algorithm for simulating a shear-strain step test. [Color figure can be viewed in the online issue, which is available at wileyonlinelibrary.com.]

integration scheme is implemented in the current $\mathrm{BD}$ approach, special care must be taken when choosing the time step. To guarantee acceptable convergence to the central values (relative errors lower than $4 \%$ ) in strong flow-induced regimes (typical of the shear-strain step imposed for starting a relaxation test in this work) a time step $\Delta t=\left(\zeta a^{2} / k_{B} T\right) \cdot 10^{-5}$ is the minimal requirement.

\section{SHEAR RELAXATION TEST ALGORITHM}

A flowchart of the algorithm employed to predict the shear-modulus after a shear-strain step by means of BD approach is presented in Figure 3. Simulation requires a BD system ( $N$ bead-rod chain models) in thermal equilibrium before applying the material strain function. An equilibrium stage (i.e., with no external flow) is carried out until one of two criteria is satisfied: stabilization of the stored internal-bending energy or integration during three times the longest characteristic time associated with the multi bead-rod chain model. In our modeling, characteristic time is given by the rotational diffusion time of an equal-length multibead rigid-rod system containing $n$ beads ${ }^{53}$ :

$$
\lambda_{n}=\frac{\zeta L^{2} n(n+1)}{72(n-1) k_{B} T}
$$

Once an equilibrium configuration has been obtained for the BD system, a shear-strain function $\gamma=\dot{\gamma}_{0} t$ is applied. During the shear-strain step a constant shear-strain rate $\dot{\gamma}_{0}$ is imposed during a total charging time $t_{c}$ and the BD-calculated shearstress and internal bending-energy signals are stored. It is important to point out that the shearstrain at the end of the shear-strain step, i.e., $\gamma_{0}=\dot{\gamma}_{0} t_{c}$, is imposed in such a way that rheological response is independent of the maximal applied shear-strain. On the other hand, with the aim to closely mimic the rapid strain-rates imposed experimentally, the constant shear-rate implemented in the BD simulations is calculated using a shearing time shorter than the period $P$ associated with a reciprocal frequency $\omega \lambda_{n}=10^{3}$. Knowing that $P=2 \pi / \omega$, shearing time is then calculated arbitrarily as a quarter of such period:

$$
t_{c}=\frac{\pi \lambda_{n}}{2 \times 10^{3}}
$$

At the end of the charging time, shear-strain function is turned off to zero and the BD system is let to relax. BD-calculated shear-stress and internal-energy signals are stored in function of time until at least one of two criteria is satisfied: stabilization of the stored internal bending-energy during one-half of the longest characteristic time of the equivalent multibead rigid-rod model or integration during three times the same longest characteristic time.

\section{RESULTS AND DISCUSSION}

The main purpose of this article is to predict the evolution of the shear-stress of a suspended nonfreely jointed bead-rod chain with a natural bent configuration after applying a shear-strain function and compare the BD simulation results with the experimental counterpart for treated SWNTs diluted within a Newtonian solvent. Figure 1 presents the shear-stress relaxation signatures after a shear-strain step of $1 \%$, applied on two different suspensions of treated SWNT (0.1 and $0.2 \mathrm{wt} \%$ ) within an epoxy resin. Shear-strain of $1 \%$ is within the linear viscoelastic region according to the strain sweep test carried out on those two dilute treated SWNT suspensions. ${ }^{59}$ A very fast relaxation process is observed for the two suspensions, even masked by the motor response time of the rheometer. The rheological behavior is very close to that one exhibited by the solvent alone, which in fact corresponds with a completely viscous-fashion response.

Shear-strain tests in the linear regime are particularly interesting in the current case because the Newtonian suspending medium is supposed to relax almost instantaneously and, therefore, any viscoelastic character observed during the transition stage would be attributable exclusively to the dynamics of the suspended particles. In addition, evolution of the internal bending-energy [eq. (10)] can be also used to distinguish the different relaxation processes observed at intermediate frequencies in the BD simulations of small-amplitude oscillatory deformation tests and supposed to be associated with different bending modes. ${ }^{38}$ 


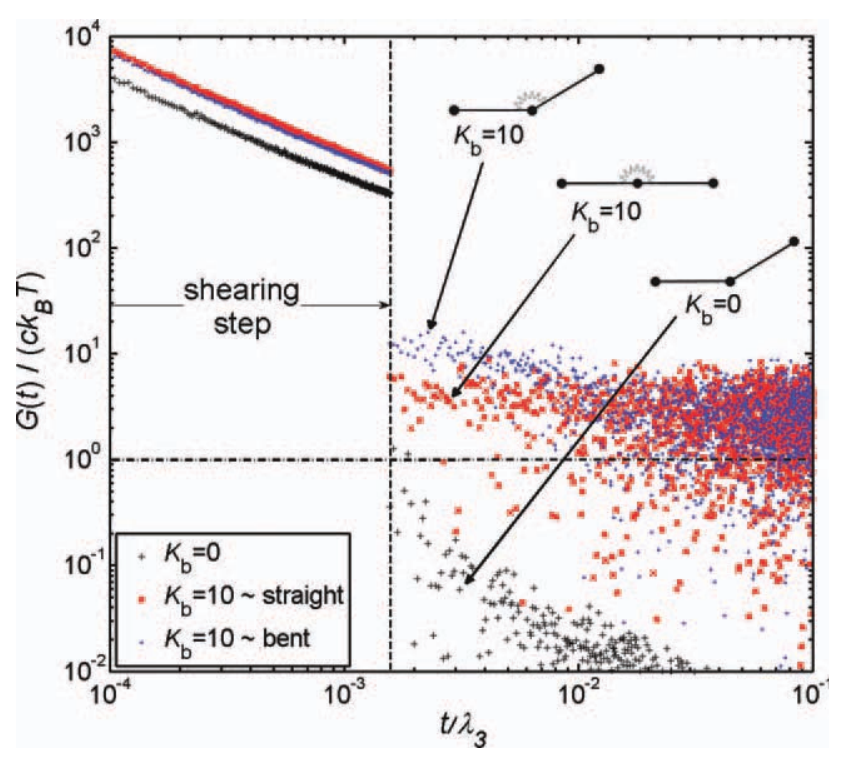

Figure $4 \mathrm{BD}$ prediction of the shear-modulus relaxation for different (three-bead)-(two-rod) chains: freely jointed, nonfreely jointed with natural straight configuration $\left(K_{b}=\right.$ $10)$ and nonfreely jointed with natural bent configuration $\left(K_{b}=10\right.$ and $\left.\Delta \theta^{\max }=30^{\circ}\right)$. Dashed vertical line marks the end of the shearing step. A total shear-strain $\gamma_{0}=1$ was imposed. $n=3, c=a=\zeta=1, k_{B} T=0.1$ are the parameters of the BD simulation. $\lambda_{3}$ is the rotational diffusion time of a (three-bead) rigid-rod system of equal length. [Color figure can be viewed in the online issue, which is available at wileyonlinelibrary.com.]

Let us to study first the relaxation behavior of the simplest bead-rod chain model, i.e., the (three-bead)(two-rod) system. Figure 4 presents the evolution of the shear-modulus after a linear shearing-step for the trimer system and two nonfreely jointed (threebead)-(two-rod) chains: (1) with straight configuration in absence of external forces and (2) with bent configuration in absence of external forces. BD simulations showed that independently of the bending rigidity constant (freely or nonfreely jointed) and the quiescent configuration (straight or bent) shear-stress vanishes practically instantaneously, like a perfect viscous liquid. A drop of the shear-modulus in almost three orders of magnitude is observed for the trimer system. On the other hand, nonfreely jointed systems showed a smaller drop of approximately two orders of magnitude. At first sight, these results seem incoherent with the presumed existence of a relaxation spectrum for the shear-stress in bead-rod chains, especially when the bead-spring counterparts exhibit neat viscoelastic relaxation behavior under the same conditions. However, testing conditions are precisely the key to understand the obtained $\mathrm{BD}$ results. In fact, as already mentioned the shearing step currently imposed is analogue with an oscillatory deformation in the high frequency range, particularly at $\omega_{R}=10^{3}$. From the BD simulation results obtained for the same bead-rod chain model in dynamic mode ${ }^{38}$ is noticed that at this solicitation frequency the loss modulus is quite larger than the storage modulus (in more than one order of magnitude) for all the systems simulated in Figure 4. In other words, below the current deformation conditions, the different (three-bead)-(two-rod) chain models have a preponderant viscous character, which is naturally manifested during the shearstrain step test by a quasi instantaneous shear-stress relaxation. In fact, shear-stress is proportional to the shear strain-rate in a viscous liquid, hence once shearing is stopped (i.e., shear strain-rate goes instantaneously to zero) shear-stress presents a dramatic discontinuity as well.

However, despite the extremely rapid relaxation of the shear-modulus, in Figure 4 is observed that the freely jointed system behaves differently with respect to the nonfreely jointed ones, especially during the relaxation stage. Moreover, in the very early relaxation times, a slight distinction can be made between the natural straight and the natural bent nonfreely jointed (three-bead)-(two-rod) chain models. Those differences in the evolution of the shearmodulus (blurred by the stochastic nature of the simulation) can be attributed to the different contributions given by the internal bending-energy modes to the dynamics of the bead-rod chain model, as conjectured before in the $\mathrm{BD}$ simulations of the same coarse-grained model under dynamic linear viscoelasticity tests. ${ }^{38}$ In fact, this hypothesis is supported also by the curves of stored bending-energy during the simulated shear-strain step tests, which offer more clear information than the shear-stress signal.

Figure 5 compares the BD predicted stored bending-energy during a shear-strain step test for the two nonfreely jointed (three-bead)-(two-rod) chains simulated in Figure 4: natural straight configuration versus natural bent configuration $\left(\Delta \theta^{\max }=30^{\circ}\right)$. A neat increment of the stored bending-energy is observed during the shearing step. At the end of the shearing step, the naturally bent system stocked 3.2 times more energy than the naturally straight system; a fact in coherence with the differences of storage modules found in the BD simulations of dynamic tests $^{38}$ and confirming the coherence of the coarsegrained model. In Figure 5, differences in the relaxation times associated with the restitution of the stored bending-energy are also appreciable. The natural bent system exhibits a relaxation time that is almost twofold that one of the natural straight model. That difference appears consistent with the larger values of shear-modulus exhibited by the natural bent system in the early relaxation times. On the other hand, in both cases, relaxation of the stored bending-energy happens in time scales quite shorter than the characteristic rotational diffusion time $\lambda_{3}$, which also explains why the relaxation 


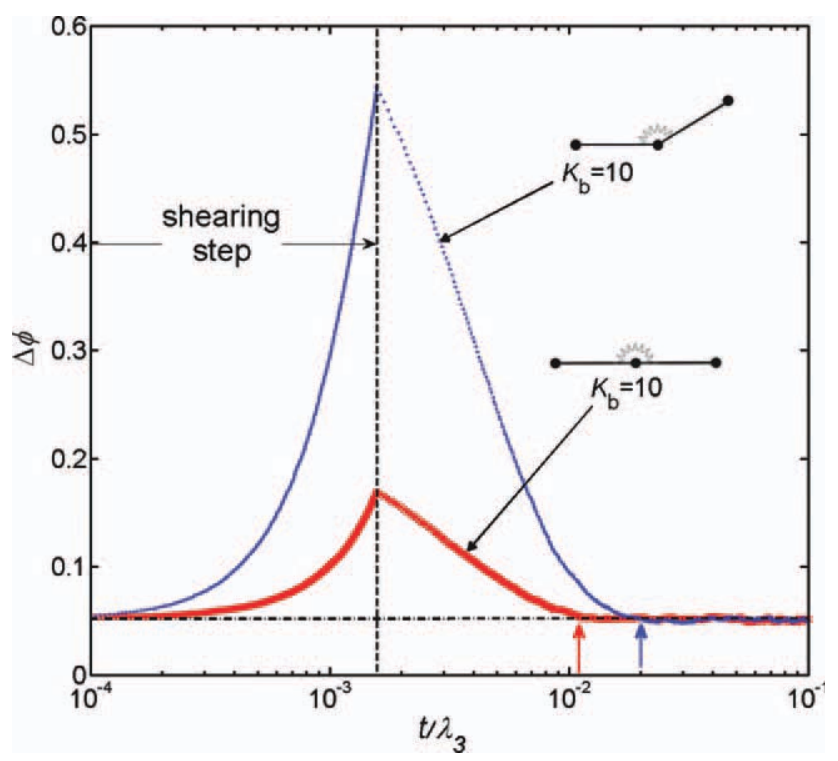

Figure $5 \mathrm{BD}$ prediction of the stored bending-energy during a shear-strain step test for two different nonfreely jointed (three-bead)-(two-rod) chains: naturally straight configuration and naturally bent configuration $\left(\Delta \theta^{\max }=\right.$ $\left.30^{\circ}\right)$. Dashed vertical line marks the end of the shearing step. A total shear-strain $\gamma_{0}=1$ was imposed. $n=3, c=a$ $=\zeta=1, k_{B} T=0.1$ are the parameters of the BD simulation. Stored bending-energy is calculated as follows: $\Delta \phi=\phi-\phi_{\min }$, where $\phi_{\min }$ is the minimal value of the bending potential energy. $\lambda_{3}$ is the rotational diffusion time of a (three-bead) rigid-rod system of equal length. [Color figure can be viewed in the online issue, which is available at wileyonlinelibrary.com.]

processes observed in the simulations of dynamic tests appear only in the high frequency range. BD simulations demonstrate again that a naturally bent SWNT structure exhibits a different dynamic behaviour in comparison with that of a perfectly straight SWNT. This fact corroborates that defects and their structural consequences must be taken into account when tackling with the dynamics of a single SWNT in suspension.

In what follows, effect of the bending rigidity constant is tackled comparing the BD simulated shearstrain step tests of three nonfreely jointed (threebead)-(two-rod) chains with bent configurations (at minimal bending-energy) and different values of bending rigidity constant. As expected, shear-modulus signature did not vary with respect to the scenario presented in Figure 4; a practically instantaneous relaxation of the shear-stress is exhibited indistinctly of the value of the bending rigidity constant. On the other hand, evolution of the stored bending-energy during the shear-strain step test is presented in Figure 6 and shows more exploitable results.

It appears clearly that the internal bending-energy stored by the nonfreely (three-bead)-(two-rod) chain model during the shearing step is related directly to the rigidity of the chain. This result is coherent with the increase of the storage modulus in the high frequency range when enhancing the bending rigidity constant $K_{b} .{ }^{38}$ Moreover, as higher is the bending rigidity shorter is the relaxation time associated with the stored bending-energy. Previous fact explains why higher input frequencies are required for activating the mild elasticity during an oscillatory deformation test as higher is the bending rigidity of the bead-rod chain.

Evolutions of the internal bending energy during a shear-strain step test for nonfreely jointed multibead-rod chains with different number of rods $(n=$ $3, n=4$, and $n=6$ ) are compared in Figure 7 . Remember that the number of rods in the bead-rod chain model is related, on one hand, to the persistence length under flow conditions and, on the other one, to the linear density of bent junctions in the SWNT structure. For a given value of the bending rigidity constant, an increase in the number of constitutive rods is traduced in an enhancement of the stored bending energy during the shearing step; an expectable result given the fact that the bendingenergy stocking capacity is associated directly with the number of flexion springs in the system.

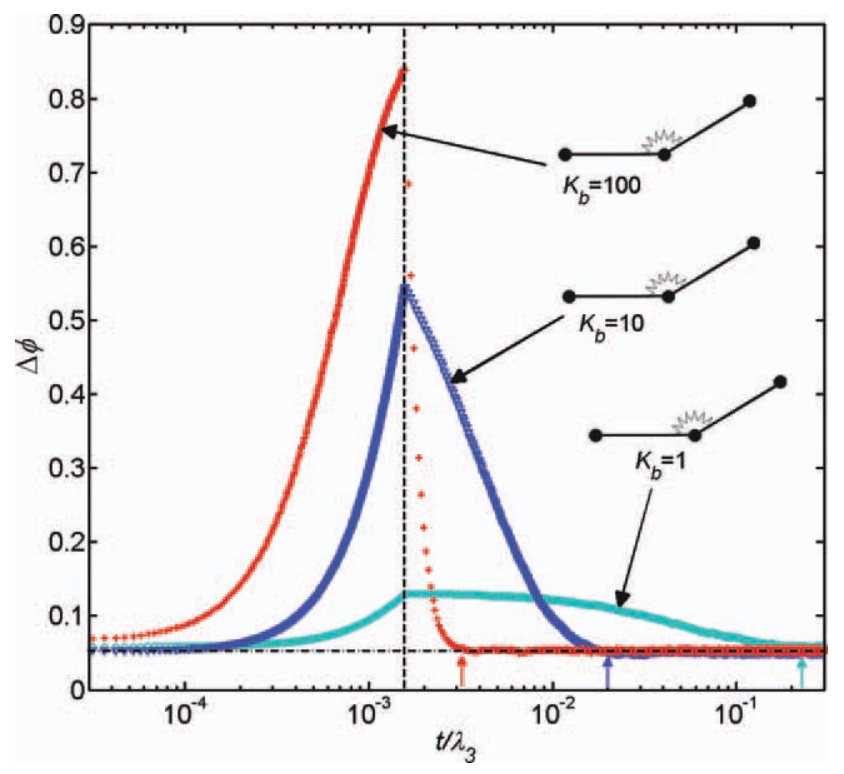

Figure $6 \mathrm{BD}$ prediction of the stored bending-energy during a shear-strain step test for three nonfreely jointed (three-bead)-(two-rod) chains naturally bent $\left(\Delta \theta^{\max }=30^{\circ}\right)$ with different values of bending rigidity constant. Dashed vertical line marks the end of the shearing step. A total shear-strain $\gamma_{0}=1$ was imposed. $n=3, c=a=\zeta=1, k_{B} T$ $=0.1$ are the parameters of the BD simulation. Stored bending-energy is calculated as follows: $\Delta \phi_{\text {shear }}=$ $\phi-\phi_{\text {eq }}$, where $\phi_{\min }$ is the minimal value of the bending potential energy. $\lambda_{3}$ is the rotational diffusion time of a (three-bead) rigid-rod system of equal length. [Color figure can be viewed in the online issue, which is available at wileyonlinelibrary.com.] 


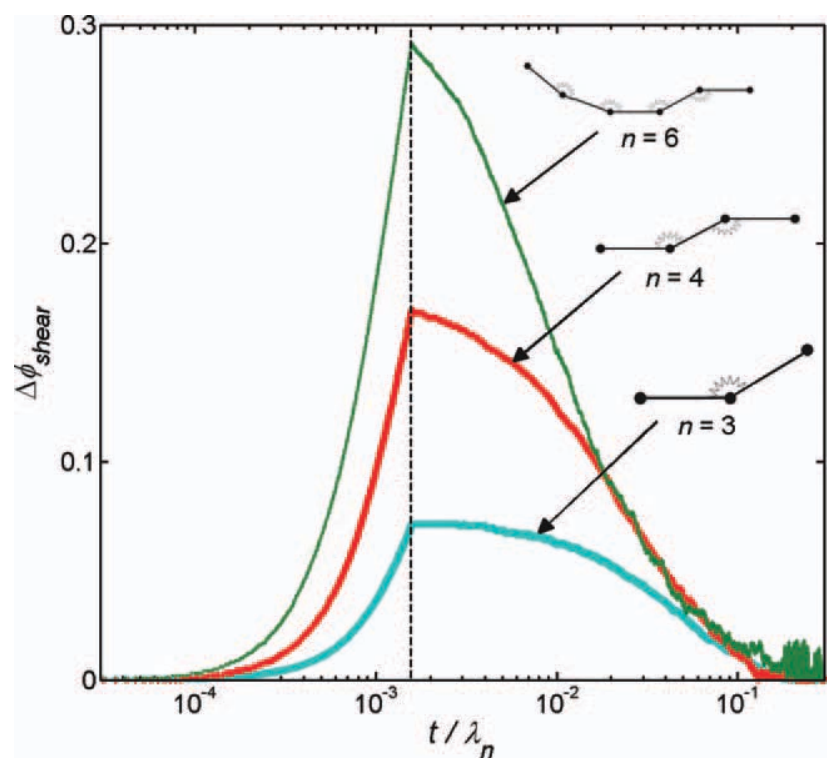

Figure 7 BD prediction of the stored bending-energy during a shear-strain step test for three nonfreely jointed bead-rod chains naturally bent $\left(\Delta \theta^{\max }=30^{\circ}\right)$ with different number of rods. Dashed vertical line marks the end of the shearing step. A total shear-strain $\gamma_{0}=1$ was imposed. $c=a=\zeta=K_{b}=1$, and $k_{B} T=0.1$ are the parameters of the BD simulation. Stored shear bending-energy is calculated as follows: $\Delta \phi=\phi-\phi_{\min }$, where $\phi_{\mathrm{eq}}$ is the bending-energy at equilibrium. $\lambda_{n}$ is the rotational diffusion time of an equivalent multibead rigid-rod system of equal length. [Color figure can be viewed in the online issue, which is available at wileyonlinelibrary.com.]

Moreover, the different energy-relaxation curves obtained in function of the number of rods are in coherence with the different curves of complex modulus simulated elsewhere for bead-rod chains with different number of rods. ${ }^{38}$ Figure 7 reveals also that the time scale of the different bending-relaxation modes is related directly to the number of constitutive rods in the chains (note that Figure 7 is plotted in a reduced time scale); a fact in phase with the increase of the frequency range associated with the mild elastic response when larger number of constitutive rods are considered during the oscillatory dynamical tests of dilute suspensions of bead-rod chains. $^{38}$

Finally, with the aim of evaluate the real performance of the nonfreely jointed bead-rod chain model for mimicking the structure of a SWNT, the experimental shear-stress evolution after a shear-strain step applied on a $0.1 \mathrm{wt} \%$-treated SWNTs suspension (in Figure 1) is compared with the predicted shear-stress evolution from a BD simulation fed with real physical parameters. A physical bead friction coefficient can be estimated based on the physical parameters of the HiPCO treated SWNTs $(L=400$ $700 \mathrm{~nm}$, diameter $d=0.8-1.3 \mathrm{~nm})^{52,60,61}$ and the suspending medium (Newtonian epoxy resin with an apparent viscosity of $10 \mathrm{~Pa} \mathrm{~s}^{-1}$ ) used in the shear-strain step tests presented in Figure 1. This physical bead friction coefficient depends on the number of centres of hydrodynamic resistance (number of beads $n$ ) as shown elsewhere. ${ }^{38}$ For example, a SWNT ( $L=400 \mathrm{~nm}, d=1 \mathrm{~nm})$ mimicked by a four-rods $(n=5)$ chain model has a physical bead friction coefficient $\zeta_{b}=1.67 \times 10^{-6} \mathrm{~Pa} \cdot \mathrm{m} \cdot \mathrm{s}$. Theoretically, in absence of external forces, persistence length $L_{p}$ relates the bending rigidity constant $K_{b}$ and the thermal factor $k_{B} T$ as follows: $L_{p}=K_{b} / k_{B} T$. Persistence length in HiPCO-treated SWNT has been determined experimentally between 26 and $138 \mu \mathrm{m}$ by using a semiflexible filament model, where the filament has a perfect tubular structure. ${ }^{52}$ We swept the experimental range of persistence lengths in our BD simulations and we identified that the best fitting was obtained with the highest value of persistence length (i.e., $L_{p}=138 \mu \mathrm{m}$ ). Authors suggest that this fact can be associated with a local stiffing effect of the SWNT structure coming from the bent junction (defect).

Experimental shear-modulus plotted in Figure 8 corresponds to the rheological response of a $0.1 \mathrm{wt}$ $\%$ SWNT suspension. Predicted shear modulus has been calculated as the sum of two contributions: one coming from the Newtonian solvent $G_{s}$ and other

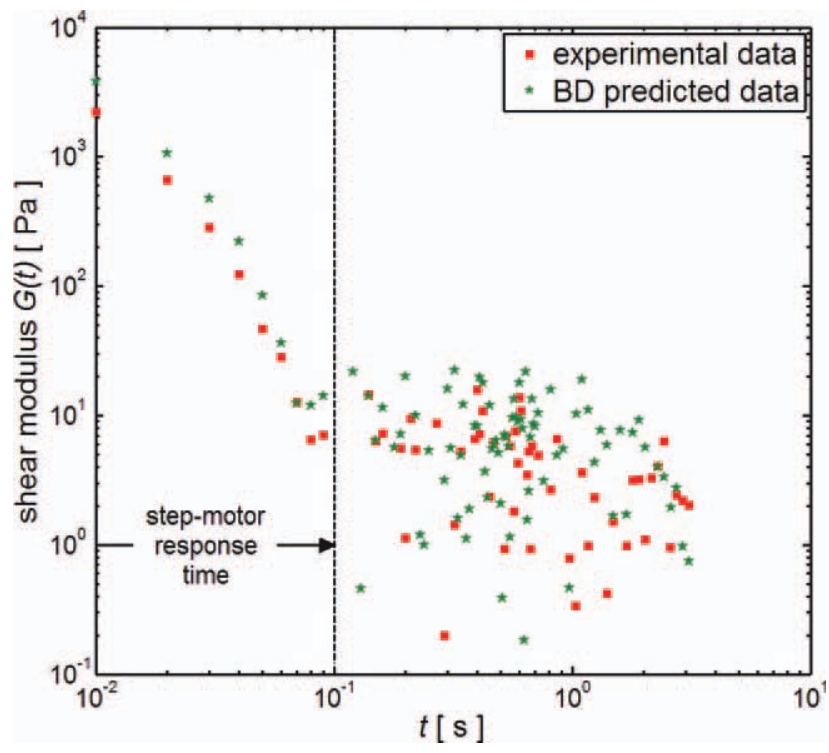

Figure 8 Comparison between the experimental shear modulus relaxation (from Fig. 1) and the BD-predicted shear modulus relaxation of a $0.1 \mathrm{wt} \%$ SWNT suspended within a preepoxy resin. A BD population of 5000 nonfreely jointed (five-beads)-(four-rods) chains monodisperse in length $(400 \mathrm{~nm})$ has been employed. Each SWNT has been modeled as a flexible bead-rod chain with an effective persistence length of $138 \mu \mathrm{m}$ and a bead friction coefficient equal to $1.67 \times 10^{-6} \mathrm{~Pa} \cdot \mathrm{m} \cdot \mathrm{s}$. Angles of bent junctions (defects) have been restricted to be lower than $30^{\circ}$. Both experimental and simulated shear-strain step data are given at $25^{\circ} \mathrm{C}$. [Color figure can be viewed in the online issue, which is available at wileyonlinelibrary.com.] 
coming from the suspended SWNTs $G_{p}$; in other words $G=G_{s}+G_{p}$. Shear modulus of the Newtonian solvent has been measured experimentally (Fig. 1). Shear modulus coming from the SWNTs has been BD-simulated. To quantify the SWNT contribution we need to estimate the number of density $c$ in a 0.1 wt \% suspension. Supposing a SWNT population monodisperse in length and diameter, the number of entities per volume $c$ can be calculated as follows:

$$
c \equiv \frac{4 \phi}{\pi d^{2} L}
$$

where $\phi$ is the volumetric fraction of SWNT in the suspension. Volumetric fraction of SWNT has been estimated by using a SWNT density equal to $1.45 \mathrm{~g}$ $\mathrm{mL}^{-1}$ (close-packed density for $[7,8]$ SWNTs $^{60}$ ) and an epoxy density equal to $1.16 \mathrm{~g} \mathrm{~mL}^{-1}$ (according to the supplier).

In spite of the $\mathrm{BD}$ simulation overestimates the evolution of the shear modulus during the early times after the shearing step (0.01-0.06 s), a direct comparison is only feasible after the step-motor response time (marked by a vertical dashed line in Fig. 8), which guarantees absolute confidence on the values recovered experimentally by the rheometer. In that context, the window of comparison is restricted to the data since $0.1 \mathrm{~s}$ (i.e., points to the right of the vertical dashed line in Fig. 8). Interestingly, after the motor response time, BD-prediction and experimental data are statistically comparable.

It is important to point out that the BD-simulated shear-modulus in Figure 8 has been obtained for a SWNT population supposed monodisperse in contour and persistence length $(400 \mathrm{~nm}$ and $138 \mu \mathrm{m}$, respectively). Nonnegligible effects of the contour length on the BD-simulated shear-modulus relaxation can be presumed in front of the results presented in Figure 7. Consideration of polydisperse SWNT populations is an important perspective of the current modeling and constitutes a work in progress.

In general, the absence of a measurable viscoelastic effect after the linear shearing of dilute SWNT suspensions appears in coherence with the quasi-instantaneous BD-simulated relaxation of the shear-stress of a nonfreely jointed bead-rod chain model in dilute solution. In other words, proposed nonfreely jointed bead-rod chain model with natural bent configuration for mimicking the structure of a treated SWNT remains consistent, at least, within the regime of linear viscoelasticity.

Based on such coherence of the BD modeling with the experimental evidence for dilute suspensions of treated SWNTs within a Newtonian solvent, identification of an equivalent micromechanical model can be envisaged taken into account the BD-simulated linear viscoelastic responses for two different material functions: a small-amplitude oscillatory and a shear-strain step. Deduction of mentioned equivalent micromechanical model is a work in progress.

\section{CONCLUSIONS}

Individual treated SWNTs in suspension are modeled as semiflexible filaments with an intrinsic bent configuration in absence of external forces considering the natural existence of topological defects on the honeycomb-like CNT wall structure. Continuous semiflexible filament model is discretized as a nonfreely jointed bead-rod chain with a nonstraight configuration in absence of external forces for simulating in $\mathrm{BD}$ the evolution of the shear stress after a linear shearing step applied on SWNT dilute suspensions within a Newtonian solvent. Number of constitutive rods in the coarse-grained model is related to the persistence length under flow conditions and the linear density of topological defects forming bent junctions in the SWNT structure.

BD-simulated shear-stress evolutions after a linear shear-strain step were coherent with the rheological responses in dynamic tests obtained before, ${ }^{38}$ proving the consistency of the nonfreely jointed bead-rod chain model with natural bent configuration in the framework of linear viscoelasticity. In all the simulations carried out, a quasi-instantaneous relaxation of the shear-stress is verified at the end of the shearing step. This viscous-like response is coherent with the nonzero limiting viscosity obtained in the dynamic response at high frequencies. Evolutions of the stored bending-energy during the simulations of shear-strain step tests are found in phase with the relaxation processes observed in the dynamic response at intermediate-high frequencies. An increment of the bending rigidity constant is traduced in an enhancement of the stored bending energy during the shearing step, a fact explaining the enhancement of the storage modulus in the high frequency range. Additionally, relaxation time of the stored bending energy after a shear-strain step shortens as the bending rigidity constant increases. Last fact is in coherence with the $\mathrm{BD}$ simulations of dynamic tests, in which the solicitation frequency required to activate the mild elasticity increases as the bending rigidity constants increases as well.

Interestingly, despite the fact that a nonnegligible elastic response is observed under dynamic oscillatory solicitations of SWNT dilute suspensions, such an elastic character is very difficult to reveal under shear relaxation tests as confirmed by the BD simulations and the experiments. Real shear-strain step tests on dilute and semidilute suspensions of treated SWNTs within an epoxy resin exhibit a quasi-instantaneous relaxation of shear stress; a behavior in 
agreement with the BD-simulated shear-strain step tests on dilute solutions of nonfreely jointed beadrod chains with natural bent configuration.

Authors express their thanks to Dr. Anson Ma (Rice University) and Prof. Malcolm Mackley (University of Cambridge) for good discussions and for providing helpful experimental data (Fig. 1). The authors also thank the GeM institute (Institute de Recherche en Génie Civil et Mécanique) at Nantes (France) for providing the computational resources.

\section{References}

1. Ruoff, R. S.; Qian, D.; Liu, W. K. Physique 2003, 4, 903.

2. Krishnan, A.; Dujardin, E.; Ebbesen, T. W.; Gibson, J. M. Phys Rev B 1998, 58, 14013.

3. Yu, M. F.; Files, B. S.; Arepalli, S.; Ruoff, R. S. Phys Rev Lett $2000,84,5552$

4. Yu, M. F.; Lourie, O.; Dyer, M. J.; Moloni, K.; Kelly, T. F.; Ruoff, R. S. Science 2000, 287, 637.

5. Pop, E.; Mann, D.; Wang, Q.; Goodson, K.; Dai, H. Nano Lett 2006, 6, 96 .

6. Wei, B.; Vajtai, R.; Ajayan, P. Appl Phys Lett 2001, 79, 1172.

7. Hong, S.; Myung, S. Nat Nanotechnol 2007, 2, 207.

8. Saito, S. Science 1997, 278, 77.

9. Postma, H. W. C.; Teepen, T.; Yao, Z. Grifoni, M.; Dekker, C. Science 2001, 293, 76.

10. Dai, H.; Hafner, J. H.; Rinzler, A. G.; Colbert, D. T.; Smalley, R. E. Nature 1996, 384, 147.

11. Kong, J.; Franklin, N. R.; Zhou, C.; Chapline, M. G.; Peng, S.; Cho, K.; Dai, H. Science 2000, 287, 622.

12. Shim, M.; Kam, N. W. S.; Chen, R. J.; Li, Y.; Dai, H. Nano Lett 2002, 2, 285

13. Kam, N. W. S.; Jessop, T. C.; Wender, P. A.; Dai, H. J Am Chem Soc 2004, 126, 6850.

14. Andrews, R.; Jacques, D.; Rao, A. M.; Rantell, T.; Derbyshire, F.; Chen, Y.; Chen, J.; Haddon, R. C. Appl Phys Lett 1999, 75, 1329.

15. Mitchell, C. A.; Bahr, J. L.; Arepalli, S.; Tour, J. M.; Krishnamoorti, R. Macromolecules 2002, 35, 8825.

16. Abdel-Goad, M.; Potschke, P. J Non-Newton Fluid 2005, 128, 2.

17. Yuen, S.; Chen-Chi, M.; Wu, H.; Kuan, H.; Chen, W.; Liao, S.; Hsu, C.; Wu, H. J Appl Polym Sci 2007, 103, 1272.

18. Kharchenko, S. B.; Douglas, J. F.; Obrzut, J.; Grulke, E. A.; Migler, K. B. Nat Mater 2004, 3, 564.

19. Moniruzzaman, M.; Winey, K. I. Macromolecules 2006, 39, 5194.

20. Hobbie, E. K. Rheol Acta 2010, 49, 323.

21. Rahatekar, S. S.; Koziol, K. K. K.; Butler, S. A.; Elliott, J. A.; Shaffer, M. S. P.; Mackley, M. R.; Windle, A. H. J Rheol 2006, 50, 599.

22. Ma, A. W. K.; Chinesta, F.; Mackley, M. R. J Rheol 2009, 53, 547.

23. Fan, Z.; Advani, S. G. Polymer 2005, 46, 5232.

24. Hinch, E. J.; Leal, L. G. J Fluid Mech 1972, 52, 683.

25. Petrie, C. J. S. J Non-Newton Fluid Mech 1999, 87, 369.

26. Sepehr, M.; Carreau, P. J.; Grmela, M.; Ausias, G.; Lafleur, P. G. J Polym Eng 2004, 24, 579.

27. Rajabian, M.; Dubois, C.; Grmela, M. Rheol Acta 2005, 44, 521.

28. Advani, S. G.; Tucker, C. L. J Rheol 1990, 34, 367.

29. Larson, R. G. The Structure and Rheology of Complex Fluids; Oxford University Press: New York, 1999.
30. Xu, J.; Chatterjee, S.; Koelling, K. W.; Wang, Y.; Bechtel, S. E. Rheol Acta 2005, 44, 537.

31. Hough, L. A.; Islam, M. F.; Janmey, P. A.; Yodh, A. G. Phys Rev Lett 2004, 93, 168102-1.

32. Montesi, A.; Morse, D. C.; Pasquali, M. J Chem Phys 2005, 122, 084903.

33. Davis, V. A.; Ericson, L. M.; Parra-Vasquez, A. N. G.; Fan, H.; Wang, Y.; Prieto, V.; Longoria, J. A.; Ramesh, S.; Saini, R. K.; Kittrell, C.; Billups, W. E.; Adams, W. W.; Hauge, R. H.; Smalley, R. E.; Pasquali, M. Macromolecules 2004, 37, 154.

34. Rai, P. K.; Parra-Vasquez, N. G.; Chattopadhyay, J.; Pinnick, R. A.; Liang, F.; Sedana, A. K.; Hauge, R. H.; Billups, W. E.; Pasquali, M. J Nanosci Nanotechnol 2007, 7, 3378.

35. Rahatekar, S. S.; Koziol, K. K.; Kline, S. R.; Hobbie, E. K.; Gilman, J. W.; Windle, A. H. Adv Mater 2009, 21, 874.

36. Morse, D. C. Macromolecules 1998, 31, 7030.

37. Shankar, V.; Pasquali, M.; Morse, D. C. J Rheol 2002, 46, 1111.

38. Cruz, C.; Illoul, L.; Chinesta, F.; Régnier, G. Rheol Acta 2010, $49,1141$.

39. Lijima, S.; Ichihashi, T.; Ando, Y. Nature 1992, 356, 776.

40. Dimitrakopulos, G. P.; Dravid, D. P.; Karakostas, T. H.; Pond, R. C. Acta Crystallogr A 1997, 53, 341.

41. Ajayan, P. M.; Ravikumar, V.; Charlier, J. Phys Rev Lett 1998, $81,1437$.

42. Han, J.; Anantram, M. P.; Jaffe, R. L.; Kong, J.; Dai, H. Phys Rev B 1998, 57, 14983.

43. Lambin, P. H.; Meunier, V. Appl Phys A 1999, 68, 263.

44. Wako, K.; Oda, T.; Tachibana, M.; Kojima, K. Jpn J Appl Phys 2008, 47, 6601.

45. Xue, B.; Shao, X.; Cai, W. J Chem Theory Comput 2009, 5, 1554.

46. Binh, V. T.; Vincent, P.; Feschet, F.; Bonard, J. J Appl Phys 2000, 88, 3385.

47. Clauss, W. Appl Phys A Mater 1999, 69, 275.

48. Ouyang, M.; Huang, J.; Cheung, C. L.; Lieber, C. M. Science 2001, $291,97$.

49. Vijayaraghavan, A.; Marquardt, C. W.; Dehm, S.; Hennrich, F.; Krupke, R. Carbon 2010, 48, 494.

50. Lamprecht, C.; Danzberger, J.; Lukanov, P.; Tilmaciu, C. M.; Galibert, A. M.; Soula, B.; Flahaut, E.; Gruber, H. J.; Hinterdorfer, P.; Ebner, A.; Kienberg, F. Ultramicroscopy 2009, 109, 899.

51. Tsyboulski, D. A.; Bachilo, S. M.; Weisman, R. B. Nano Lett 2005, 5, 975.

52. Fakhri, N.; Tsyboulski, D. A.; Cognet, L.; Weisman, R. B.; Pasquali, M. Proc Natl Acad Sci USA 2009, 106, 14219.

53. Bird, R. B.; Curtiss, C. F.; Armstrong, R. C.; Hassager, O. Dynamics of Polymer Liquids: Kinetic Theory; Wiley Inter-Science: New York, 1987; Vol.2.

54. Liu, T. W. J Chem Phys 1989, 90, 5826.

55. Somasi, M.; Khomami, B.; Woo, N. J.; Hur, J. S.; Shaqfeh, E. S. G. J Non-Newton Fluid Mech 2002, 108, 227.

56. Doyle, P. S.; Shaqfeh, E. S. G.; Gast, A. P. J Fluid Mech 1997, 334, 251.

57. Grassia, P.; Hinch, E. J. J Fluid Mech 1996, 308, 255.

58. Hassager, O. J Chem Phys 1974, 60, 4001.

59. Ma, W. K. A. The Microstructure and Rheology of Carbon Nanotube Suspensions. PhD Thesis, University of Cambridge, Cambridge, UK, 2008.

60. Parra-Vasquez, A. N. G.; Stepanek, I.; Davis, V. A.; Moore, V. C.; Haroz, E. H.; Shaver, J.; Hauge, R. H.; Smalley, R. E.; Pasquali, M. Macromolecules 2007, 40, 4043.

61. Mendes, M. J.; Schmidt, H. K.; Pasquali, M. J Phys Chem B $2008,112,7467$. 